\title{
Association between Hepato-Biliary Status and HbA1C in Type 2 Diabetes Mellitus with Coronary Artery Disease (CAD)
}

\author{
R. Ramesh, N. AdhishwarKumaran, V. KuzhandaiVelu, R. Reeta, M. SathishBabu, \\ G. Niranjan \\ Department of Biochemistry, MGMCRI, SBV University, Pilliyarkuppam, Pondicherry, India \\ Email: Kuzhandai2707@gmail.com
}

Received 26 February 2015; accepted 28 April 2015; published 4 May 2015

Copyright (C) 2015 by authors and Scientific Research Publishing Inc.

This work is licensed under the Creative Commons Attribution International License (CC BY). http://creativecommons.org/licenses/by/4.0/

\section{(c) (7) Open Access}

\begin{abstract}
Bilirubin has both antioxidant activity and cardio-protective potential. Decreased levels are associated with predicting CAD. Recent studies showed that bilirubin has inverse correlation with glycemic status. So in this we evaluated the role of Hepato-biliary function as a marker of predictor CAD in patient with T2DM. Total 100 subjects included 50 T2DM patients with CAD and 50 T2DM without CAD. All the subject venous blood was collected after overnight fasting for biochemical analysis. After the statistical analysis, the results showed significant difference between two groups as follows total bilirubin $(0.32 \pm 0.15 ; 0.90 \pm 032 ; p=0.000)$, direct bilirubin, indirect bilirubibn, Total Protein, Albumin, ALT, AST, HbA1c. Our finding implies that decreased serum bilirubin increases the risk of CAD in patients with T2DM and it shows inverse correlation between HbA1c and bilirubin.
\end{abstract}

\section{Keywords}

Bilirubin, Coronary Artery Disease, Type 2 Diabetes Mellitus

\section{Introduction}

India is diabetic capital of the world over a decade and now it is heading to lead in Cardiovascular Disease also as both diabetes and coronary artery disease (CAD) shares several risk factors in common. Patients with Diabetes Mellitus (DM) are at four to five fold increased risk to CAD. Type 2 Diabetes Mellitus (T2DM) are commonly found in association with Non Alcoholic Fatty Liver Disease (NAFLD) [1], the mechanisms of liver injury in T2DM involve insulin resistance (IR), oxidative stress, apoptosis and adipokines [2]. IR results in 
peripheral lipolysis and upregulates hepatic de novo lipogenesis. This increase in lipogenesis and lipid peroxidation, and decrease in adiponectin which is protective adipokines make T2DM patients with NAFLD, vulnerable to Coronary Artery Disease (CAD) [3]. The Framingham risk score analysis conducted by Iognnou et al. showed that there exists an association between elevated serum alanine amino transferase (ALT) and 10 years of CAD [4]. Previous studies have demonstrated that circulating levels of ALT, aspartate aminotransferase (AST) and gamma-glutamyltransferase (GGT) are increased in individuals with IR and Metabolic Syndrome [5] [6]. Increased levels of GGT found to be associated with various atherosclerotic risk factors in T2DM and literatures are suggesting that De Ritis ratio (AST/ALT) could be used to assess the extent of hepto-biliary damage [7] [8]. Recent reviews state that bilirubin prevents lipid and lipoprotein oxidation and hence protects against CVD. Serum bilirubin is inversely related to CAD and also increases in case of myocardial infarction [9] [10].

The oxidative stress contributed by hepatic steatosis in T2DM alters endothelial function and leads to decreased production of nitric oxide which may also contribute to the atherogenic effect of NAFLD in T2DM [11]. The NAFLD is also associated with increased free radical production as a result of alteration in iron metabolism and increased activation of Fenton's reaction. This may further increase in glycation of various proteins including hemoglobin. Hence our study is planned in order to assess the status of hepatobiliary function in CAD patients with T2DM and their correlation with glycemic status.

\section{Methodology}

Patients who visited the Department of Cardiology and General medicine OPD at Mahatma Gandhi Medical College and Research Institute and those whose are diagnosed as CAD was included in the study. Then the subjects was divided into two groups by randomize sampling:Group 1 (Subjects)—Type 2 Diabetes Mellitus patients with confirmed CAD and Group 2 (Control)-Type 2 Diabetes Mellitus patients without CAD. The subject with chronic kidney disease, chronic hepatic disease, bypass surgery or percutaneous coronary intervention, chronic or current infections, presence of the thalassemia trait and any other hemoglobin disorder, any systemic disease that could cause high bilirubin concentrations, neoplastic disease and autoimmune disease where excluded from the study.

After appropriate screening total 100 subjects (50 subjects and 50 controls) was included in the study between age group 30 to $60 \mathrm{yrs} .3 \mathrm{ml}$ of blood was collected from the patient and the samples was processed for glucose, fasting plasma insulin, HbA1c, serum bilirubin, total protein, albumin, AST and ALT estimation using IFCC approved methods in fully automated chemistry analyzer. To find the significant difference in liver function test and glycemic status between two groups Student's t Test was used and correlation analysis was performed to assess the association between bilirubin and glycemic status among groups. All these statistical analysis was performed by SPSS 20 software.

\section{Results}

The average age of the control (T2DM without CAD) and subject (T2DM with CAD) included in the study. Table 1 shows the mean and standard of the glycemic status of both groups. The mean fasting plasma glucose of T2DM with CAD group (160 mg/dl) has higher than T2DM without CAD (98.81 mg/dl). HbA1c levels also showed statistically significant difference between the two groups. The plasma insulin levels were lower in T2DM patients with CAD than without. Insulin resistance was more pounced in group who compared to group 2.

The hepatobiliary status of both groups is depicted in Table 2. The study (Figure 1) showed that bilirubin level were low in T2DM patient with CAD $(0.32 \pm 0.15)$ when compared with T2DM patients without CAD $(0.90 \pm 0.32)$. T2DM patients with CAD showed low levels of total protein and albumin when compared to T2DM patient without CAD. Liver enzymes like AST and ALT were increased significant in T2DM patient when compared to T2DM patient without CAD. De-ritis ratio also showed significant high in subjects (2.75 \pm $0.68)$ and in control (1.44 \pm 0.46$)$. Correlation analysis revealed an inverse relationship between bilirubin and glycemic indicator HbA1c and HOMAir with $r$ value -0.334 and -0.360 respectively.

The Table 3 depicts the odds ratio of altered hepatobiliary function associated with CAD in T2DM patients. The odds of decreased bilirubin level associate with subjects (odds ratio: 2.15) is about two times more when compared with controls. The odds that increased ALT are associates with T2DM with CAD is 2.7 time more than T2DM without CAD. 
Table 1. Glycemic status in T2DM Patients with and without CAD.

\begin{tabular}{|c|c|c|c|}
\hline Parameters & $\begin{array}{c}\text { Group } 1 \\
\text { Type } 2 \text { DM without CAD } \\
(\mathrm{n}=50) \\
\text { Mean } \pm \text { SD }\end{array}$ & $\begin{array}{c}\text { Group } 2 \\
\text { Type } 2 \text { DM with CAD } \\
(\mathrm{n}=50) \\
\text { Mean } \pm \text { SD }\end{array}$ & “p” Value \\
\hline Fasting Plasma Glucose (mg/dl) ${ }^{*}$ & $98.81 \pm 28.12$ & $160 \pm 54.28$ & 0.000 \\
\hline HbA1c (\%)* & $7.14 \pm 1.73$ & $8.90 \pm 1.97$ & 0.001 \\
\hline Insulin (uIU/L) ${ }^{*}$ & $6.37 \pm 1.35$ & $8.62 \pm 2.18$ & 0.000 \\
\hline Insulin Resistance (HOMAir) ${ }^{*}$ & $1.61 \pm 0.14$ & $3.41 \pm 1.26$ & 0.000 \\
\hline
\end{tabular}

*"p" Value is significant at the 0.001 level.

Table 2. Hepato-biliray status in T2DM Patients with and without CAD.

\begin{tabular}{|c|c|c|c|}
\hline Parameters & $\begin{array}{c}\text { Group } 1 \\
\text { Type } 2 \text { DM without CAD } \\
(n=50) \\
\text { Mean } \pm \text { SD }\end{array}$ & $\begin{array}{c}\text { Group } 2 \\
\text { Type } 2 \text { DM with CAD } \\
(n=50) \\
\text { Mean } \pm \text { SD }\end{array}$ & "p" Value \\
\hline Total Protein (g/dl) ${ }^{*}$ & $7.62 \pm 0.46$ & $6.89 \pm 1.10$ & 0.002 \\
\hline Albumin (g/dl) ${ }^{*}$ & $4.43 \pm 0.90$ & $3.71 \pm 0.62$ & 0.001 \\
\hline Globulin (g/dl) & $3.19 \pm 1.03$ & $3.18 \pm 0.58$ & 0.949 \\
\hline $\mathrm{A} / \mathrm{G}$ ratio & $1.18 \pm 0.15$ & $1.08 \pm 1.20$ & 0.669 \\
\hline $\operatorname{AST}(\mathrm{U} / \mathrm{L})^{*}$ & $28.79 \pm 7.87$ & $90.69 \pm 36.64$ & 0.000 \\
\hline $\operatorname{ALT}(\mathrm{U} / \mathrm{L})^{*}$ & $22.55 \pm 13.30$ & $34.42 \pm 17.27$ & 0.006 \\
\hline De ritis ratio $(\mathrm{AST} / \mathrm{ALT})^{*}$ & $1.44 \pm 0.46$ & $2.75 \pm 0.68$ & 0.000 \\
\hline Total Bilirubin (mg/dl) ${ }^{*}$ & $0.90 \pm 0.32$ & $0.32 \pm 0.15$ & 0.000 \\
\hline Direct Bilirubin (mg/dl) ${ }^{*}$ & $0.28 \pm 0.1$ & $0.12 \pm 0.05$ & 0.000 \\
\hline Indirect Bilirubin (mg/dl) ${ }^{*}$ & $0.60 \pm 0.23$ & $0.20 \pm 0.12$ & 0.000 \\
\hline
\end{tabular}

*"p" Value is significant at the 0.001 level.

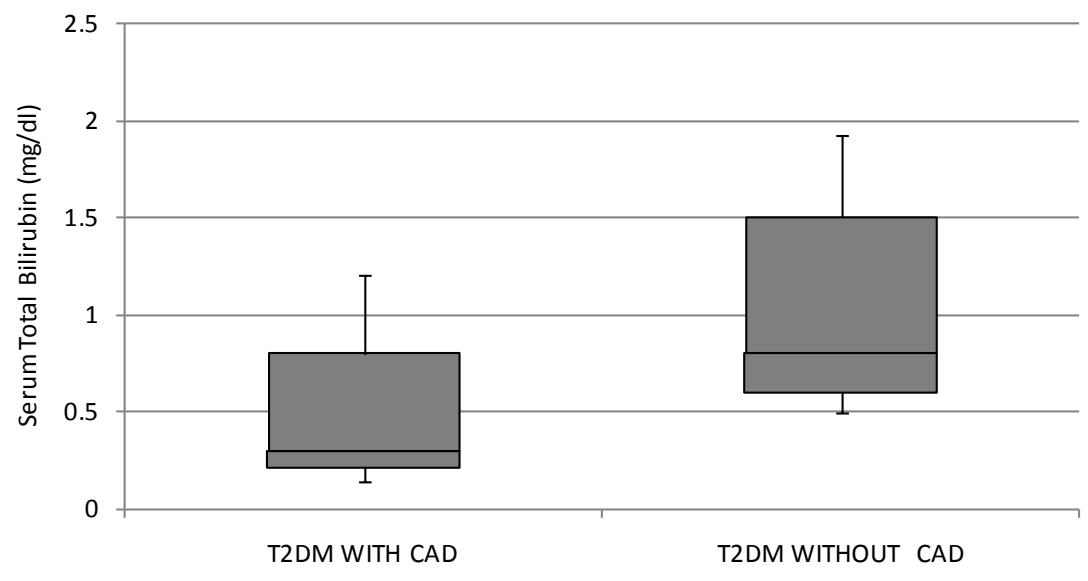

Figure 1. The distribution of serum bilirubin in type 2 diabetes mellitus patients with and without coronary artery disease.

\section{Discussion}

Bilirubin acts as a cardioprotective agent by scavenging lipid preoxides and other products of physiological oxidation. Schwether et al. in 1994 showed that plasma bilirubin concentration inversely correlated with CAD [12]. Masood et al. and Hopkin et al. also showed low level of bilirubin in CAD patients when compared with 
Table 3. Odds ratio for ALT, De-ritis ratio and Serum Bilirubin.

\begin{tabular}{ccc}
\hline Parameters & Odds ratio & $\mathbf{0 . 9 5}$ confidence Interval \\
\hline ALT & 2.75 & $0.27-2.61$ \\
De ritis ratio & 0.31 & $0.03-3.16$ \\
Serum Bilirubin & 2.15 & $0.36-1.27$ \\
\hline
\end{tabular}

normal Subjects [13]. Our result was also in concodence with the above mentioned studies and showed low levels of bilirubin in T2DM patient with CAD when compared with to T2DM without CAD. A survey conducted by National Health and Nutrition Examination from 1999-2004 reported that increased bilirubin levels are associated with reduced prevalence of peripheral artery disease [14].

In our study we also compared total protein and albumin levels between the two groups and found their serum levels were decreased in T2DM patient with CAD [15]. De-ritis ratio which is used as surrogate marker for identifying non-alcoholic fatty liver disease was increased among T2DM with CAD when compared with T2DM without CAD [16]. Some other studies have also reported that NALFD and insulin resistance was an independent risk for CAD. The association between insulin resistance and NAFLD had already been established in many studies [1] [17]-[19]. The link between low serum bilirubin levels and CAD in T2DM can be explained by the increased oxidative stress associated with both the pathogenesis of T2DM and CAD.

Present Study also showed an inverse correlation between HbA1c, Insulin resistance and serum bilirubin. This was in concordance with published results of Mayer et al. and Oda et al. which showed a negative correlation between HbA1c and cardiovascular risk factors [9] [20].

\section{Conclusion}

The study concluded that decreased level of serum bilirubin and increased HbA1c increases the risk factor for CAD in patient with T2DM. There is elevation of liver enzyme also seen in T2DM with CAD.

\section{Limitation of the Study}

The major limitation of this study is lesser number of participants. This was due to the lack of time as this was the ICMR short term student project. In this study, we could have compared the Intima media thickness of the coronary artery with serum biliurbin levels which could have been made serum biliurbin as a better maker.

\section{Acknowledgements}

We would like to acknowledge ICMR for accepting this project as a Short Term Student project of Mr. N. Adhishwar Kumaran

\section{References}

[1] Treeprasertsuk, S., Lopez-Jimenez, F. and Lindor, K.D. (2011) Nonalcoholic Fatty Liver Disease and the Coronary Artery Disease. Digestive Diseases and Sciences, 56, 35-45. http://dx.doi.org/10.1007/s10620-010-1241-2

[2] Kotronen, A., Juurinen, L., Tiikkainen, M., Vehkavaara, S. and Yki-Järvinen, H. (2008) Increased Liver Fat, Impaired Insulin Clearance, and Hepatic and Adipose Tissue Insulin Resistance in Type 2 Diabetes. Gastroenterology, 135, $122-$ 130. http://dx.doi.org/10.1053/j.gastro.2008.03.021

[3] Edmison, J. and McCullough, A.J. (2007) Pathogenesis of Non-Alcoholic Steatohepatitis: Human Data. Clinics in Liver Disease, 11, 75-104. http://dx.doi.org/10.1016/j.cld.2007.02.011

[4] Treeprasertsuk, S., Leverage, S., Adams, L.A., Lindor, K.D., St. Sauver, J. and Angulo, P. (2012) The Framingham Risk Score and Heart Disease in Nonalcoholic Fatty Liver Disease. Liver International, 32, 945-950. http://dx.doi.org/10.1111/j.1478-3231.2011.02753.x

[5] Kuzhandai velu, V., Jyothirmayi, B. and Kumar, J.S. (2011) Insulin Resistance and Alanine Amino Transaminase (ALT) Levels in First Degree Relatives of Type 2 Diabetes Mellitus. Diabetes \& Metabolic Syndrome: Clinical Research \& Reviews, 5, 143-147. http://dx.doi.org/10.1016/j.dsx.2012.02.011

[6] Gopal, N., Selvam, A. and Muddegowda, P.H. (2012) Serum Gamma Glutamyl Transferase levels in Obese South Indian Adults with Reference to Atherogenic Lipid Risk Factors and Lipid Peroxides. International Journal of Medical 
and Health Sciences, 1, 35-42.

[7] Majhi, S., Baral, N., Lamsal, M. and Mehta, K.D. (2006) De Ritis Ratio as Diagnostic Marker of Alcoholic Liver Disease. Nepal Medical College Journal, 8, 40-42.

[8] Arthi, M., Niranjan, G., Hanifah, M. and Srinivasan, A.R. (2011) Efficacy of De Ritis Ration in Diagnosing Liver Diseases in Puducherry Population. Advance Laboratory Medicine International, 1, 61-68.

[9] Mayer, M. (2000) Association of Serum Bilirubin Concentration with Risk of Coronary Artery Disease. Clinical Chemistry, 46, 1723-1727.

[10] Arumalla, V.K., Gopal, N. and Bn, R. (2012) Role of Serum Bilirubin in Coronary Artery Disease. International Journal of Medical and Health Sciences, 1, 56-62.

[11] Cersosimo, E. and DeFronzo, R.A. (2006) Insulin Resistance and Endothelial Dysfunction: The Road Map to Cardiovascular Diseases. Diabetes/Metabolism Research and Reviews, 22, 423-436. http://dx.doi.org/10.1002/dmrr.634

[12] Schwertner, H.A., Jackson, W.G. and Tolan, G. (1994) Association of Low Serum Concentration of Bilirubin with Increased Risk of Coronary Artery Disease. Clinical Chemistry, 40, 18-23.

[13] Hopkins, P.N., Wu, L.L., Hunt, S.C., James, B.C., Vincent, G.M. and Williams, R.R. (1996) Higher Serum Bilirubin Is Associated with Decreased Risk for Early Familial Coronary Artery Disease. Arteriosclerosis, Thrombosis, and Vascular Biology, 16, 250-255. http://dx.doi.org/10.1161/01.ATV.16.2.250

[14] Perlstein, T.S., Pande, R.L., Beckman, J.A. and Creager, M.A. (2008) Serum Total Bilirubin Level and Prevalent LowerExtremity Peripheral Arterial Disease: National Health and Nutrition Examination Survey (NHANES) 1999 to 2004. Arteriosclerosis, Thrombosis, and Vascular Biology, 28, 166-172. http://dx.doi.org/10.1161/ATVBAHA.107.153262

[15] Stocker, R., Yamamoto, Y., McDonagh, A.F., Glazer, A.N. and Ames, B.N. (1987) Bilirubin Is an Antioxidant of Possible Physiological Importance. Science, 235, 1043-1046. http://dx.doi.org/10.1126/science.3029864

[16] Kawamoto, R., Kohara, K., Kusunoki, T., Tabara, Y., Abe, M. and Miki, T. (2012) Alanine Aminotransferase/Aspartate Aminotransferase Ratio Is the Best Surrogate Marker for Insulin Resistance in Non-Obese Japanese Adults. Cardiovascular Diabetology, 11, 117. http://dx.doi.org/10.1186/1475-2840-11-117

[17] Balkau, B. and Eschwège, E. (1999) Insulin Resistance: An Independent Risk Factor for Cardiovascular Disease? Diabetes, Obesity and Metabolism, 1, S23-S31. http://dx.doi.org/10.1046/j.1463-1326.1999.0010s1023.x

[18] Misra, V.L., Khashab, M. and Chalasani, N. (2009) Non-Alcoholic Fatty Liver Disease and Cardiovascular Risk. Current Gastroenterology Reports, 11, 50-55. http://dx.doi.org/10.1007/s11894-009-0008-4

[19] Hamaguchi, M., Kojima, T., Takeda, N., Nagata, C., Takeda, J., Sarui, H., Kawahito, Y., Yoshida, N., Suetsugu, A., Kato, T., Okuda, J., Ida, K. and Yoshikawa, T. (2007) Nonalcoholic Fatty Liver Disease Is a Novel Predictor of Cardiovascular Disease. World Journal of Gastroenterology, 13, 1579-1584. http://dx.doi.org/10.3748/wjg.v13.i10.1579

[20] Oda, E. and Kawai, R. (2011) Bilirubin Is Negatively Associated With Hemoglobin A1c Independently of Other Cardiovascular Risk Factors in Apparently Healthy Japanese Men and Women. Circulation Journal, 75, 190-195.

http://dx.doi.org/10.1253/circj.CJ-10-0645 